\title{
Um Relato de Experiência da Aplicação do SPIDe no Ensino de IHC
}

\author{
Beatriz Rêgo, Filipe Garrido, Jean Rosa, Iuri Santos, Ecivaldo Matos \\ Departamento de Ciência da Computação - Universidade Federal da Bahia (UFBA) \\ Av. Adhemar de Barros, s/n - Salvador - BA - Brasil \\ \{beatrizbr, filipe.garrido, jean.rosa, iuri.santos, ecivaldo\}@ufba.br
}

\begin{abstract}
This paper presents a SPIDe aplication experience with undergraduate students in Human-Computer Interaction classes in Federal University of Bahia (UFBA). The application goal was present the SPIDe to students through practical activities, promoting engagement and collaboration in interaction design process. The results were satisfactory allowed us to evaluate modifications in future SPIDe applications.
\end{abstract}

Resumo. Este artigo descreve a experiência de aplicação do SPIDe (Semio-Participatory Interaction Design Framework) com alunos de graduação da disciplina de Interação Humano-Computador da Universidade Federal da Bahia (UFBA). O objetivo foi apresentar o framework e realizar uma atividade prática com os estudantes da disciplina, visando promover $o$ engajamento e colaboração no processo de concepção do design de interação. Os resultados, satisfatórios, nos permitiram avaliar modificações em futuras aplicações do SPIDe.

\section{Introdução}

A comunicabilidade é definida como a habilidade de sistemas computacionais interativos de comunicarem-se com os seus usuários [Barbosa e Silva 2010]. O usuário consegue entender por meio da interface para que o sistema serve, a quem ele se destina, quais suas vantagens, como o sistema funciona e quais são as possibilidades de interação [Raquel e Barbosa 2007].

O conceito de comunicabilidade foi proposto por De Souza (2005), criadora da teoria de Interação Humano-Computador (IHC) intitulada Engenharia Semiótica (EngSem). Segundo a EngSem, a interação é um processo de comunicação entre o designer de interação e o usuário por meio do preposto do design (interface) [De Souza 2005]. Todavia, quando trata-se do processo de Design de Interação, a EngSem não possui métodos para auxiliar o designer de interação [Lamas e Pender, 2014].

Segundo Lowgren (2014), o design de interação é o processo de modelagem das coisas digitais para o uso humano. Nesse sentido, Rosa (2016) fez uma leitura da definição de Lowgren (2014) sob a ótica da EngSem. Para o pesquisador, o design de interação é um processo composto pela construção do diálogo entre o designer de interação e os (potenciais) usuários (i.e. modelagem da comunicação). Todavia, conforme Rosa (2016), o designer de interação não deve estar centrado somente na 
VI Congresso Brasileiro de Informática na Educação (CBIE 2017)

Anais dos Workshops do VI Congresso Brasileiro de Informática na Educação (WCBIE 2017)

comunicação, mas estender ao contexto de uso do software e as necessidades dos (potenciais) usuários.

De acordo com Baranauskas (2013), o design de interação deve ser um processo social e que não deve incluir somente o designer de interação, mas também os usuários, saindo da perspectiva do fazer para em direção ao fazer com. Para isso, a pesquisadora sugere que os usuários sejam participantes e coautores da interação produzida a partir do uso de técnicas participativas [Baranauskas, 2013].

Considerando o papel participativo do design de interação sob a perspectiva da EngSem, Rosa e Matos (2016) construíram um processo para o Design de Interação chamado de SPIDe (Semio-Participatory Interaction Design). O SPIDe foi construído a partir da compreensão do conceito de Design Semioparticipativo desenvolvido por Baranauskas (2013).

O objetivo desse artigo é apresentar um relato de experiência da aplicação do SPIDe em duas turmas da disciplina de Interação Humano-Computador (MATC-72) da Universidade Federal da Bahia no semestre letivo de 2017.1. Esse artigo é organizado da seguinte forma: a seção 2 apresenta o SPIDe; posteriormente, a seção 3 apresenta à aplicação do SPIDe nas turmas de IHC; a seção 4 retrata a análise observacional da aplicação do SPIDe; e por fim, as considerações finais do artigo são apresentadas na seção 5 .

\section{SPIDe}

A associação entre abordagens semióticas e práticas participativas para o Design de Interação é chamada por Baranauskas (2013) de Design Semioparticipativo. Para a pesquisadora, o Design Semioparticipativo considera o estudo da interação/comunicação por meio da participação do usuário no processo de design. Essa construção parte do entendimento da comunicação como um processo social bem definido e os artefatos mediadores dessa comunicação devem endossar o design, fazendo sentido para os interlocutores (i.e. designers e usuários).

Do ponto de vista da Baranauskas (2013), no design de interação a Semiótica pode auxiliar na compreensão do processo e dos elementos da comunicação que existe entre designer-usuário. Por sua vez, ao fazer uso do Design Participativo, é possível atribuir ao usuário o papel de codesign, contribuindo de forma efetiva em todo o processo de design de interação. Todavia, vale salientar que o uso do Design Participativo não é simplesmente colocar o usuário em contato com o designer, mas criar condições e incentivar para que o designer e o usuário, no papel de codesginer, atuem em conjunto.

Partindo do pressuposto de Design Semioparticipativo, o SPIDe vem sendo desenvolvido no Grupo de Pesquisa e Extensão em Informática, Educação e Sociedade Onda Digital $^{1}$ [Rosa, 2016; Rosa e Matos, 2016; Pita et al., 2017]. O SPIDe foi concebido inicialmente para o uso em softwares educacionais considerando aspectos multiculturais escolares [Rosa, 2016; Rosa e Matos, 2016]. Para Rosa e Matos (2015), a interação (humano-computador) do software educacional deve considerar para além dos

\footnotetext{
${ }^{1}$ http://www.ondadigital.ufba.br/
} 
VI Congresso Brasileiro de Informática na Educação (CBIE 2017)

Anais dos Workshops do VI Congresso Brasileiro de Informática na Educação (WCBIE 2017)

aspectos imediatos da interação, mas contemplar a diversidade sociocultural, atendendo também à questões pedagógicas.

Nesse sentido, para guiar o processo de design de interação semioparticipativo proposto pelo SPIDe, sua composição inicial teve como prática guiadora o Design Centrado na Comunicação [Rosa, 2016; Rosa e Matos, 2016]. O Design Centrado na Comunicação (DCC) [Barbosa, Paula e Lucena 2004] é uma prática para o design de interação baseada na EngSem. Por meio dela, é possível projetar a interação (humano-computador) como uma conversa entre designer e usuário por meio da interface. O DCC divide o processo de design de interação em três fases: análise de contexto, engenharia de interface e avaliação da nova interação [Barbosa, Paula e Lucena 2004].

Baseados nessas etapas do DCC, Rosa e Matos (2016) selecionaram técnicas do Design Participativo que pudessem proporcionar a participação de estudantes do Ensino Fundamental (i.e. crianças), professores e designers. As seguintes técnicas compuseram a versão inicial do SPIDe: contextual inquiry, brainstorm, braindraw e think-aloud (vide Figura 1).

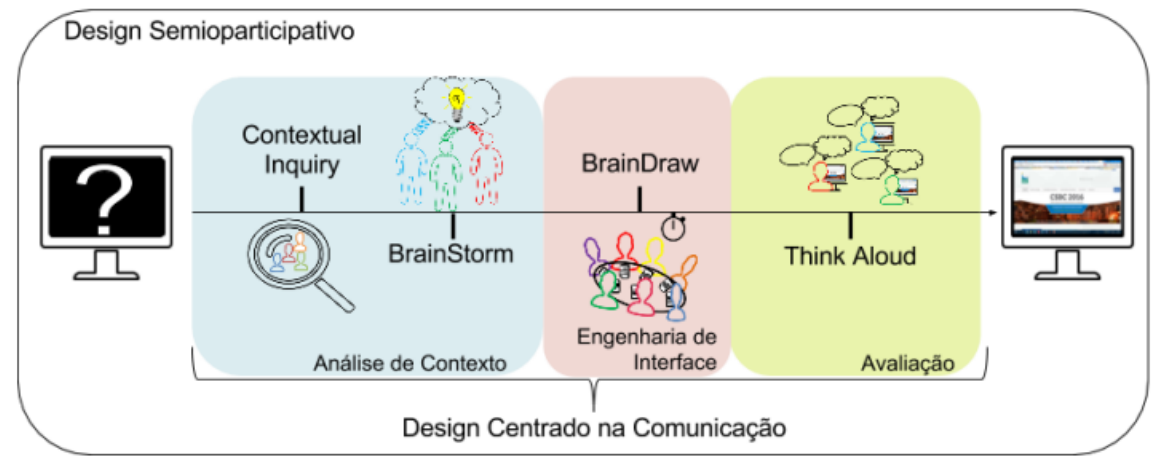

Figura 1. SPIDe [Rosa e Matos, 2016]

A análise de contexto tem por objetivo conhecer o sujeito-usuário, seu contexto sociocultural, o contexto do uso do software e como ele resolve seus problemas laborais. Para atingir esses objetivos, Rosa e Matos (2016) propuseram o uso das técnicas contextual inquiry (cf. [Muller, Haslwanter e Dayton, 1997]) e brainstorm (cf. [Faste et al., 2013]). A partir da análise de contexto o sujeito-usuário tem voz ativa, podendo fornecer diretrizes sobre como a solução pode ser construída com críticas e sugestões de melhoria da resolução de suas atividades a partir do auxílio de uma tecnologia computacional interativa [Rosa e Matos, 2016].

Por sua vez, a fase de engenharia de interface é o momento onde são gerados protótipos de interação. Nessa fase, segundo Rosa e Matos (2016), todos os participantes (usuários e designers) constroem colaborativamente a interface do artefato computacional. Para essa construção os participantes dispõem na interface signos que fazem parte do seu contexto sociocultural. Para que isso ocorra colaborativamente, os pesquisadores propuseram estabelecer o braindraw como técnica. Conforme Muller, Haslwanter e Dayton (1997) o braindraw propicia a construção de desenhos da interface a partir da fusão das ideias do usuário. Isso faz com que a interface tenha uma natureza diversificada, segundo a visão dos sujeitos-participantes. Ao término do desenho, os 
VI Congresso Brasileiro de Informática na Educação (CBIE 2017)

Anais dos Workshops do VI Congresso Brasileiro de Informática na Educação (WCBIE 2017)

participantes selecionam democraticamente um único desenho e os designer devem transformá-lo em um protótipo.

Por fim, a avaliação consiste na aplicação da técnica think-alound. Em que, o participante verbaliza seus pensamentos durante a interação com a interface produzida. Isso possibilita que o designer/avaliador perceba as principais reações durante a experiência do participante no uso do artefato. Áudio e vídeo dessa interação deve ser gravado para examinação posterior [Rosa e Matos, 2016].

Em um estudo posterior, Pita et al. (2017), em uma pesquisa inicial, adaptaram o SPIDe para a participação de sujeitos-usuários cegos e/ou com deficiência visual. Pita et al. (2017), a partir de uma pesquisa-ação identificaram que alguns participantes de sua pesquisa não sentiam-se confortáveis durante a aplicação do contextual inquiry e por isso sugeriram a inclusão da técnica storytelling na composição do SPIDe, possibilitando que de modo inconsciente o sujeito-usuário optasse pela aplicação de uma das técnicas. A Figura 2 apresenta a nova versão do SPIDe com a introdução do storytelling na análise de contexto.

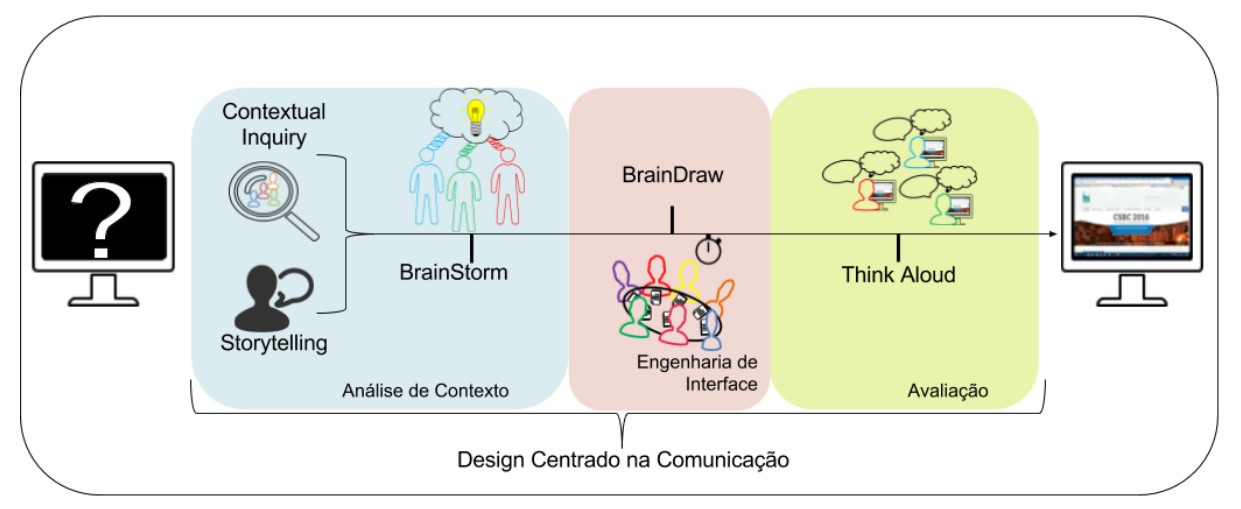

Figura 2. SPIDe após a adaptação realizada por Pita et al. (2017).

\section{Aplicação do SPIDe}

A aplicação do SPIDe ocorreu em duas turma da disciplina de IHC da UFBA. As turmas são compostas por alunos de graduação nos cursos de Licenciatura em Computação, Bacharelado em Sistemas de Informações, nesses cursos a disciplina é obrigatória, e outros cursos que possuem IHC como disciplina optativa: Bacharelado em Ciência da Computação e Engenharia da Computação.

A Tabela 1 apresenta o perfil dos participantes da aplicação do SPIDe através da distribuição da quantidade de alunos por curso de graduação na universidade. Vale também destacar que a Turma-2 da disciplina IHC possui 1 aluno de outra universidade sediada na cidade de Salvador que está a cursar a disciplina de IHC como aluno-visitante.

Além dos estudantes, participaram da aplicação do SPIDe o professor da disciplina e três pesquisadores, sendo eles dois pesquisadores-observadores, um pesquisador-mediador. Os pesquisadores-observadores são mestrandos e 0 pesquisador-mediador doutorando em Ciência da Computação, na linha de pesquisa de Interação Humano-Computador e Informática na Educação. 
VI Congresso Brasileiro de Informática na Educação (CBIE 2017)

Anais dos Workshops do VI Congresso Brasileiro de Informática na Educação (WCBIE 2017)

Foram propostos dois problemas, um para cada turma. O primeiro (Turma-1) consistiu em elencar soluções computacionais para otimizar e auxiliar o treino de usuários em academias de musculação, sem a necessidade de manipular a tela do smartphone durante o treino. O segundo problema, aplicado na Turma-2, consistiu em conceber um software educacional online que propicie a reunião de alunos em grupos de estudos, similares aos grupos de revisão criados entre os alunos presencialmente.

Tabela 1. Perfil dos alunos nas turmas de IHC.

\begin{tabular}{|c|c|c|}
\hline Curso & Turma-1 & Turma-2 \\
\hline Bacharelado em Ciência da Computação & 11 & 10 \\
\hline Engenharia da Computação & 2 & 0 \\
\hline Licenciatura em Computação & 1 & 0 \\
\hline Bacharelado em Sistemas de Informação & 0 & 13 \\
\hline Visitante & 0 & 1 \\
\hline
\end{tabular}

Por trata-se de duas turmas com 14 e 24 participantes, respectivamente ( $c f$. Tabela 1), número acima do previsto pela abordagem do SPIDe, não foi aplicada a técnica contextual inquiry, bem com o storytelling. Assim, o pesquisador-mediador acabou por descrever verbalmente o cenário e a história necessária para $o$ prosseguimento das etapas (simulando o storytelling). Por outro lado, ainda não foi possível realizar a fase de avaliação do SPIDe. Sendo assim, foi realizado aplicado com os estudantes o storytelling (simulado pelo pesquisador-mediador), o brainstorm e o braindraw. A Figura 3 apresenta as notas autoadesivas pós-brainstorm.

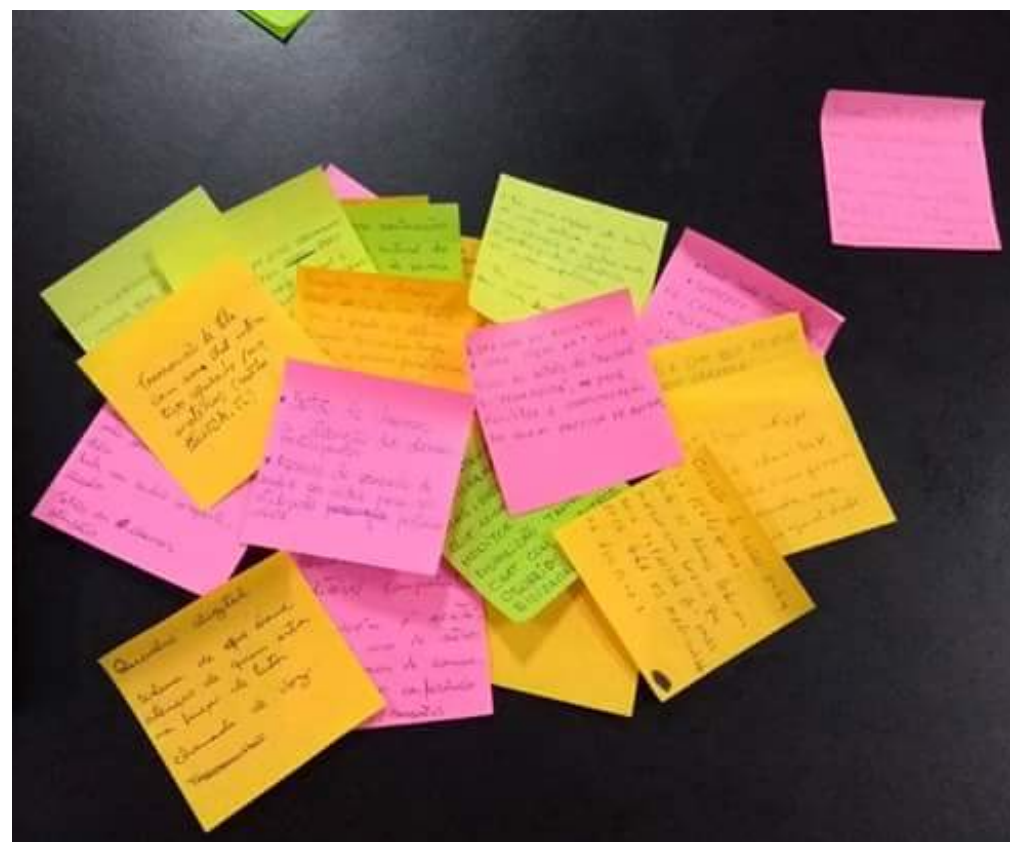

Figura 3 - Resultados do brainstorm 
VI Congresso Brasileiro de Informática na Educação (CBIE 2017)

Anais dos Workshops do VI Congresso Brasileiro de Informática na Educação (WCBIE 2017)

Ao término das duas aplicações, uma em cada turma, foram apresentados os resultados de cada grupo. Descrevendo-se os pontos fortes, fracos, semelhantes e divergentes entre cada proposta final, confrontando com as sugestões do brainstorm para verificar se a solução criada (protótipo de baixa fidelidade) contemplava as funcionalidades identificadas por eles. Por sua vez, os pesquisadores e o professor fizeram relatórios da aplicação.

\section{Observações da aplicação do SPIDe}

As observações feitas pelos pesquisadores (observadores/mediador) e pelo professor da disciplina durante a aplicação do SPIDe foram divididas em subseções, descrevendo os eventos ocorridos em cada uma das turmas separadamente.

\subsection{Turma-1}

Os pesquisadores-observadores percebeu que a Turma-1 ficou motivada durante a aplicação do brainstorm. Para resolver o problema proposto para a Turma-1, os estudantes propuseram a criação de um smartwatch e o uso de fones de ouvido para auxiliar o treino na academia de musculação ( $c f$. Figura 4).

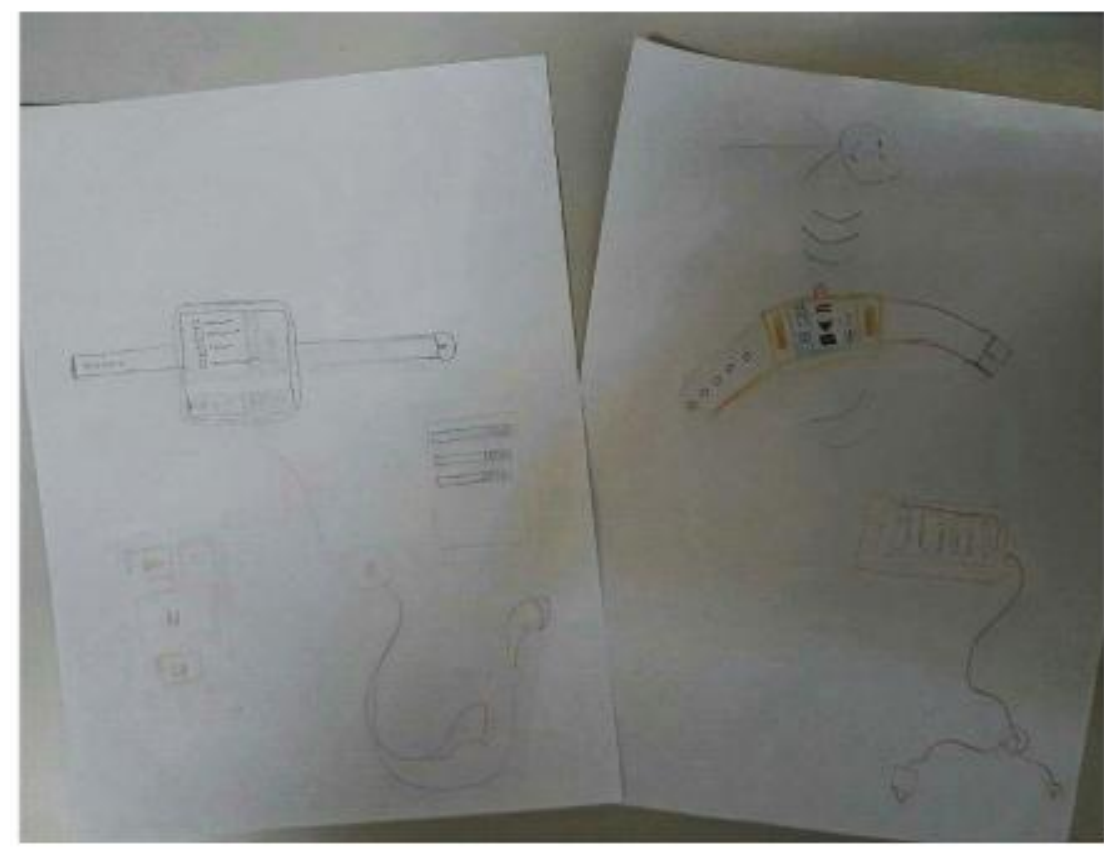

Figura 4 - Protótipo de baixa fidelidade problema 1

Nota-se que na folha do lado esquerdo da Figura 4, pode ser visto que o fone de ouvido está conectado no smartwatch. No desenho à direita, o fone de ouvido encontra-se conectado ao smartphone. Esses protótipos foram discutidos com os alunos em sala, ressaltando que o problema descrito procurava promover a facilidade de uso, que poderia ser comprometida com o fone conectado a pulseira.

Após o levantamento dessa discussão, os alunos sugeriram fones com conexão bluetooth. Mas durante a discussão com o pesquisador-mediador, os estudantes responderam que os fones conectados por fio eram dispositivos conhecidos de sua 
VI Congresso Brasileiro de Informática na Educação (CBIE 2017)

Anais dos Workshops do VI Congresso Brasileiro de Informática na Educação (WCBIE 2017)

realidade. Isso demonstra a influência sociocultural durante a produção dos desenhos no braindraw.

No brainstorm algumas ideias acabaram por se repetir, sugerindo que a quantidade de pessoas podem ter ocasionado as similaridades por conta das conversas paralelas. Os aplicadores notaram a dificuldade dos alunos em repassar o papel no qual estavam desenhando na técnica braindraw. Pois os alunos tinha receio de repassar o papel sem terem concluído o desenho que tinham se proposto a realizar. Segundo um dos pesquisadores-observadores, isso pode ter acontecido devido a falta de compreensão de como a técnica braindraw funcionava, para o pesquisador-mediador, isso acontecia por causa do preciosismo dos estudantes em finalizar sua ideia.

\subsection{Turma-2}

A Turma-2, diferente do comportamento apresentado pela Turma-1, interagiu com o pesquisador-mediador desde o primeiro momento. Demonstrando interesse em criar uma solução para o problema apresentado, um ambiente educacional online similar aos grupos de estudo presenciais.

Os alunos relacionaram objetos "reais" do seu convívio, transformando em ideias para a solução do problema. Representando os objetos pela técnica braindraw, o exemplo da proposta de solução mais comum na resolução do problema foi sugerido a utilização de um "quadro digital" (ver Figura 5), fazendo alusão ao modo como as monitorias acontecem.

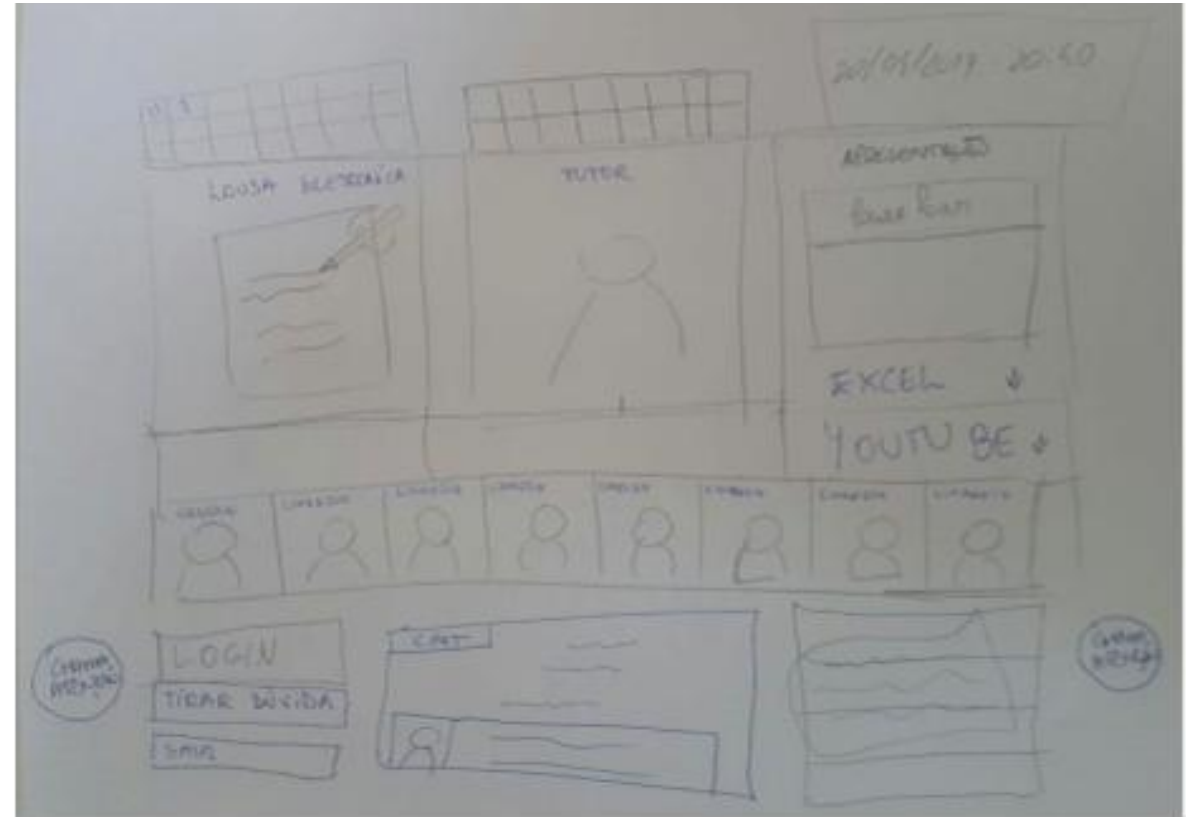

Figura 5 - Protótipo de baixa fidelidade problema 2

Outras sugestões foram: o uso de um botão que informe qual aluno deseja realizar um questionamento, evitando conflito de comunicação; a criação de uma ordem na sequência de fala dos usuários no ambiente interativo; e a agregação de diversas ferramentas externas no ambiente (ver Figura 5). 
Na Figura 5 também pode ser visto a possibilidade de apresentação de slides (indicada pela ferramenta Power Point) e link com o YouTube. Essas opções fazem referência a ferramentas de suporte que são utilizadas cotidianamente pelos alunos como forma de aprendizado extra classe. Com isso, foi possível notar que os participantes fizeram uso das suas experiências anteriores para construir um novo ambiente digital educacional.

\section{Considerações Finais}

Este trabalho apresentou o relato da experiência da aplicação do SPIDe em duas turmas (duas sessões de aplicação) da disciplina de Interação Humano Computador (IHC) da UFBA com a participação de 38 alunos de graduação com a finalidade de avaliar a capacidade técnica do framework no desenvolvimento de design de sistemas interativos centrado no usuário e com a participação efetiva dos mesmos.

O SPIDe possibilitou que os alunos participassem e aprendessem a utilizar o SPIDe visando aumentar a qualidade do design de sistemas interativos. Por fim, os alunos puderam constatar como se dá o processo de codesign e compreender a importância da inclusão do usuário em tempo de design.

A aplicação do SPIDe nas turmas possibilitou identificar aspectos culturais que estão presentes no cotidiano dos alunos, reforçando um dos principais aspectos apontado por Rosa e Matos (2016). Isto demonstra a potencialidade do SPIDe no que se diz respeito a influência de aspectos culturais no design de interação. Outro fator relevante, que ocorreu durante a aplicação do braindraw, foi a motivação dos alunos na realização da atividade. A interação e a vontade de participar eram nítidas nos grupos executores dos protótipos. Essa mesma motivação foi observada nas sugestões dadas no brainstorm (Figura 3).

Um dos pontos negativos da aplicação foi ocasionado pela grande quantidade de participantes e que resultou em dispersão e conversas paralelas, que pode resultar no enviesamento de ideias, pois os alunos acabavam por perguntar aos colegas ou até olhar o que os outros estavam fazendo, em alguns momentos. A observação dos pesquisadores durante a atividade de aplicação do SPIDe colaborou com o processo de evolução do processo, identificando possíveis melhorias nas dinâmicas que envolvem essa abordagem, em especial com um grande número de participantes.

Nesse sentido, a participação de usuários com diferentes crenças, costumes e percepções culturais, é um grande desafio para o codesign. Como trabalhos futuros será realizada a fase de avaliação da interação proposta, por meio do think-aloud e entrevistas com os participantes para identificar como eles puderam compreender o SPIDe e seus resultados.

\section{Agradecimentos}

Agradecemos aos alunos participantes da pesquisa; ao Grupo de Pesquisa e Extensão em Informática, Educação e Sociedade - Onda Digital; e às agências de fomento CAPES e FAPESB pelo apoio financeiro. 
VI Congresso Brasileiro de Informática na Educação (CBIE 2017)

Anais dos Workshops do VI Congresso Brasileiro de Informática na Educação (WCBIE 2017)

\section{Referências}

Baranauskas, M. C. C. (2013). O modelo semioparticipativo de design. In Baranauskas, M. C. C.; Martins, M. C.; Valente, J. A. (Ed.) Codesign de Redes Digitais Tecnologia e Educação a Serviço da Inclusão. Penso. p. 38-66.

Barbosa, S.; Silva, B. (2010). Interação humano-computador. Elsevier Brasil.

Barbosa, S. D. J., Paula, M., e Lucena, C. (2004). Adopting a communication-centered design approach to support interdisciplinary design teams. In SE-HCI workshop at ICSE. IET.

De Souza, C. S. (2005). The semiotic engineering of human-computer interaction. MIT Press.

Faste, H.; Rachmel, N.; Essary, R; Sheehan, E.. (2013). Brainstorm, Chainstorm, Cheatstorm, Tweetstorm. In Proceedings of the SIGCHI Conference on Human Factors in Computing Systems - CHI '13.

Lamas, D.; Pender, H. L. (2014). Reflection on the role of semiotic engineering in co-design of interaction. IEEE Latin America Transactions, v. 12, p. 48-53. ISSN 1548-0992.

Lowgren, J. (2014). Interaction Design - brief intro. In The Encyclopedia of Human-Computer Interaction (2 ed.), Mads Soegaard and Rikke Friis Dam (Eds.). The Interaction Design Foundation, Aarhus, Dinamarca, Chapter 1.

Luck, R. (2003). Dialogue in participatory design. Design Studies 24, 6, p. 523-535.

Muller, M. J.; Haslwanter, J. H.; Dayton, T. (1997). Participatory Practices in the Software Lifecycle. In Helander, M.; Landauer, T. K.; Prabhu, P. (eds.). Handbook of Human-Computer Interaction. Elsevier Science. p. 255-268.

Prates, Raquel Oliveira; Barbosa, Simone Diniz Junqueira. (2007). Introdução à teoria e prática da interação humano computador fundamentada na engenharia semiótica. In: Kowaltowski \& K. Breitman (orgs.). Jornadas de atualização em informática. p. 263-326.

Pita, G. L.; Zabot, D.; Rosa, J. C. S.; Matos, E. (2017). Adapting the SPIDe to include visually impaired users in interaction design. In: Proceedings of the 16th Brazilian Symposium on Human Factors in Computer Systems. (IHC'17). no prelo.

Pita, G. (2016). Design por todos: participação de deficientes visuais no codesign de interação humano-computador. Trabalho de Conclusão de Curso - Engenharia da Computação. Universidade Federal da Bahia.

Rosa, J. (2016). Design de Interação Multicultural: Um Framework Semioparticipativo para o (re)design da Interação de Softwares Educacionais. Dissertação de Mestrado. Instituto de Matemática e Estatística. Universidade Federal da Bahia (UFBA).

Rosa, J. e Matos, E. (2016). Semio-participatory framework for interaction (re)design of education softwares. In: Proceedings of the 15th Brazilian Symposium on Human Factors in Computer Systems. (IHC'16). 